\title{
Towards a uniform analysis of any
}

\author{
Robert van Rooij
}

(C) The Author(s) 2008. This article is published with open access at Springerlink.com

\begin{abstract}
In this paper, Universal any and Negative Polarity Item any are uniformly analyzed as 'counterfactual' donkey sentences (in disguise). Their difference in meaning is reduced here to the distinction between strong and weak readings of donkey sentences. It is shown that this explains the universal and existential character of Universal- and NPI-any, respectively, and the positive and negative contexts in which they are licensed. Our uniform analysis extends to the use of any in command and permission sentences. It predicts that whereas the use of any in permission sentences is licensed and gives rise to a universal reading, it is not licensed in command sentences.
\end{abstract}

Keywords Any - Counterfactuals · Free choice $\cdot$ Negative polarity

\section{Introduction}

The word 'any' is one of the most discussed items in theories of natural language form and interpretation. What does the word mean, and why can it be used appropriately, or even grammatically, only in a very limited set of grammatical contexts? Even more challenging, perhaps, is the question whether we can give a 'uniform' enough analysis of U(niversal) any, N(egative) $\mathrm{P}$ (olarity) I(tem) any, and

R. van Rooij ( $ه)$

Institute of Logic, Language and Computation (ILLC), University of Amsterdam,

Nieuwe Doelenstrant 15, 1012 CP Amsterdam, The Netherlands

e-mail: r.a.m.vanrooij@uva.nl 
$\mathrm{F}$ (ree) $\mathrm{C}$ (hoice) any ${ }^{1}$ that can account for their differences in meaning, but still explains why these different meanings are expressed (at least in English) by the same word. In this paper I will argue in favor of an analysis of all these uses of any as 'counterfactual' donkey sentences (in disguise). ${ }^{2}$ The difference between U-any and NPI-any will be reduced to the distinction between strong and weak readings of donkey sentences. I will show that this explains the universal and existential character of U- and NPI-any, respectively, and the positive and negative contexts in which they are licensed. FC-any will be analyzed like NPI-any and its universal force will be accounted for in terms of a new performative analysis of permission sentences.

This paper is organized as follows: In Sect. 2, I will motivate and explain Kadmon and Landman's (1993) widening and strengthening analysis of any. In Sect. 3 I will explain van Rooij's (2006a) analysis of (strong readings of) counterfactual donkey sentences, and in Sect. 4 I will analyze Universal any in terms of it. Section 5 will deal with NPI-any. It will be shown that if we treat this item as a weak 'counterfactual' donkey sentence, the meaning of NPI-any will turn out to be equivalent to a standard indefinite, but one that can be used appropriately (via Kadmon and Landman's licensing conditions) almost only in negative contexts. In Sect. 6 I discuss the use of any in permission sentences and explain why FC-any is licensed in permissions, but not in commands. I conclude in Sect. 7 with briefly comparing my proposal with some others.

\section{The widening analysis of any}

Ladusaw (1979) proposed a very successful semantic characterization of the contexts in which any can be used appropriately: as far as any is a negative polarity item it can be used appropriately, or is licensed, only in downward entailing contexts. Context $X-Y$ is downward entailing (DE) iff from the truth of $X \alpha Y$ and the fact that $\llbracket \beta \rrbracket \subseteq \llbracket \alpha \rrbracket$ (where $\llbracket \alpha \rrbracket$ denotes the semantic meaning of expression $\alpha$ ) we can conclude the truth of $X \beta Y$. Thus, a context is DE iff an expression occurring in it can be replaced by a semantically stronger expression salva veritate.

Although Ladusaw's characterization of the contexts in which any can occur appropriately is quite successful, his proposal can't be the whole story. First, any sometimes has a 'universal' reading, and if it does, it can occur in a non-DE context.

\footnotetext{
$1 \mathrm{U}$-any is normally identified with FC-any. As will become clear in the rest of this paper, I believe there is good reason to separate them.

2 It will turn out that my analysis of the various uses of any does not always crucially involve counterfactual worlds. For this reason, a reviewer suggested to change terminology. I see the force of this suggestion, but I have decided to keep on using the phrase 'counterfactual', though now making use of quotation marks. The reasons for this are two: first, the standard ordering relation used to represent the meaning of counterfactual conditionals plays a crucial role in my analysis; second, I want to make clear that the proposal is built on my earlier analysis of counterfactual donkey sentences (van Rooij 2006a).
} 
(1) a. Any owl hunts mice.

b. Any farmer who owns a donkey beats it.

c. John talked to any woman who came up to him.

Second, although the DE analysis might give a reasonably good description of the contexts that license NPI-any, it does not explain why they do so and it takes the meaning of the item itself to be irrelevant.

Kadmon and Landman (1993) (henceforth K\&L) account for these problems by taking the meaning of any into account. ${ }^{3}$ They argue that an NP of the form any $C N$ should be interpreted like the corresponding indefinite $a C N$, but where the domain of quantification over which the indefinite with any ranges is wider than the domain of $a$ $C N$, and where the sole difference between NPI-any and U-any lies in the fact that the latter, but not the former, is interpreted generically. Moreover, K\&L propose an explanation for why NPI-any is licensed only in DE contexts. This proposal involves a second meaning contribution of any: the interpretation of the sentence after domain widening has to be stronger than before widening. Consider (2a) versus ( $2 b$ ):

$$
\begin{array}{lr}
\text { a. *John ate anything. } & \exists x A t e(j, x) \\
\text { b. John didn't eat anything. } & \neg \exists x A t e(j, x)
\end{array}
$$

Sentence (2a) is unacceptable, but its negation (2b) is not. Because extension of the domain over which (kind of) things John might eat would make 'John ate something' only weaker, i.e. less informative, (2a) is correctly predicted to be unacceptable. Sentence (2b), on the other hand, gets a stronger, more informative, interpretation after domain widening, and is thus predicted to be acceptable. Thus, the licensing of NPI-any in DE contexts does not have to be stipulated, but falls out as a 'theorem' of their analysis.

K\&L's widening analysis of any is attractive and seems to be just what we need for NPI-any. ${ }^{4}$ As noted by Dayal (1998), however, the proposal is more problematic for U-any. Recall that K\&L propose that any should have the same meaning as the corresponding indefinite, and gets a universal interpretation in positive contexts just in case the corresponding indefinite is read generically. Dayal notes that this is not quite right: whereas (1c) clearly has a universal interpretation, the same sentence with the corresponding indefinite, i.e. (3), does not have a generic reading.

John talked to $a$ woman who came up to him.

Moreover, while generics allow for exceptions, sentences with U-any don't. This strongly suggests that U-any should not be thought of as being a standard indefinite

\footnotetext{
3 Krifka (1995) develops a very similar approach independently. Both approaches seek to solve other problems of Ladusaw's analysis as well, but we won't bother about that in this paper. In this paper I use K\&L's analysis, although I believe that Krifka's analysis would do equally well.

$4 \mathrm{~K} \& \mathrm{~L}$ also shows that the widening + strengthening account can explain the licensing of NPI-any in some other contexts than just the DE-ones. Krifka (1995) and van Rooij (2003) show that it can explain the licensing of NPIs in questions, while Heim (1984) and van Rooij (2006a) show (in different ways) that it accounts for the occurrence of any in antecedents of counterfactual conditionals as well.
} 
that receives a generic interpretation. But this gives rise to the question how, then, to account for the universal readings of any in (1a)-(1c)? Dayal (1998) suggests to give up on K\&L's uniform analysis of any and proposes that in contrast to NPI-any, U-any denotes a universal quantifier. However, to account for the fact that (4a) has an additional 'that-can't-be-an-accident'-interpetation not shared by (4b), she claims that U-any is a special kind of quantifier: it quantifies over possible rather than actual individuals.

(4) a. Any student in Mary's class is working on NPIs.

b. Every student in Mary's class is working on NPIs.

The intuitions Dayal wants to account for are real, but one wonders whether we really have to give up on a uniform analysis of any. We here propose that this is not needed if we analyze both any's as 'counterfactual' donkeys (in disguise). ${ }^{5}$

\section{Counterfactual donkey sentences}

\subsection{Counterfactuals}

Stalnaker (1968) and Lewis (1973) gave the following well-known analysis of (counterfactual) conditional sentences represented by $\phi>\psi$ :

$\phi>\psi$ is true at $w$ if some $\phi \wedge \psi$-worlds are closer to $w$ than any $\phi \wedge \neg \psi$ worlds.

The notion 'closer $\phi$-world to $w$ than' can be explained in terms of an ordering relation on the accessible worlds (but let us assume that all worlds are accessible). The ordering relation ' $\leq_{w}$ ' between worlds is required to obey the following conditions: reflexivity, transitivity, connectedness, and strong centering. ${ }^{6}$ The intuitive meaning of $v \leq_{w} u$ is that $v$ is at least as close, or similar, to $w$ as $u$ is. Accepting the limit assumption, i.e., $\llbracket \phi \rrbracket \neq \emptyset \Rightarrow\left\{v \in \llbracket \phi \rrbracket: \forall u \in \llbracket \phi \rrbracket: v \leq_{w} u\right\} \neq \emptyset$ (or limiting our analysis to the finite case), we can reformulate the semantics of counterfactuals in terms of a selection function. Let us define a selection function $f$ in terms of the similarity relation as follows: $f_{w}(\llbracket \phi \rrbracket)=\left\{v \in \llbracket \phi \rrbracket \mid \forall u \in \llbracket \phi \rrbracket: v \leq_{w} u\right\}$. The proposition expressed by the conditional $\phi>\psi$ is now the following set of possible worlds:

$$
\llbracket \phi>\psi \rrbracket \stackrel{\text { def }}{=}\left\{w \in W: f_{w}(\llbracket \phi \rrbracket) \subseteq \llbracket \psi \rrbracket\right\}
$$

That is, $\phi>\psi$ is true in $w$ iff $\psi$ is true at every closest $\phi$-world to $w{ }^{7}$

\footnotetext{
5 For a discussion of other proposals, see Sect. 7.

${ }^{6}$ Reflexive: for all $v: v \leq_{w} v$. Transitive: for all $v, u$ and $x$ : if $v \leq_{w} u$ and $u \leq_{w} x$, then $v \leq_{w} x$. Connected: for all $v$ and $u, v \leq_{w} u$ or $u \leq_{w} v$. Strong centering: for all $v: w \neq v \Rightarrow\left(w \leq_{w} v\right.$ and $\left.v \leq_{w} w\right)$.

7 Of course, Stalnaker's analysis is still stronger, because he makes the additional assumption that for all $\llbracket \phi \rrbracket \subseteq W$ and $w \in W, f_{w}(\llbracket \phi \rrbracket)$ is always a singleton set. In terms of the similarity relation between worlds, this means that Stalnaker assumes that $<_{w}$ obeys trichotomy: for all $u, v: u<_{w} v$ or $v<_{w} u$ or $u=v$.
} 


\subsection{Donkey sentences}

Lewis and Stalnaker assumed that the meaning of a sentence can be represented adequately by a set of possible worlds. It is well known, however, that this leads to problems for the analysis of indefinites and pronouns, especially in donkey sentences. Of course, Kamp (1981) and Heim (1982) showed that we could maintain a uniform analysis of indefinites and pronouns and still get the truth conditions of donkey sentences right, while Groenendijk and Stokhof (1991) and others have demonstrated that such an analysis need not threaten compositionality if we are willing to change our static possible-world conception of the meaning of a sentence. According to the alternative dynamic view, we interpret sentences with respect to a context that is represented by a set of world-assignment pairs, and the meaning of the sentence itself can be thought of as the update of this context, where possibilities are eliminated when the sentence is false, and the assignment of the possibilities is enriched if a new variable, or discourse referent, is introduced by way of an indefinite. ${ }^{8}$ According to this analysis, the formula $\exists x[P x] \rightarrow Q x$ is predicted to be equivalent with $\forall x[P x \rightarrow Q x]$, which means that we can account for (standard) donkey sentences in a systematic and compositional way.

\subsection{Counterfactual donkeys}

In standard indicative donkey sentences it is unproblematic to assume that the conditional connective should be interpreted as material implication. But donkey sentences show up not only in indicative mood; we have counterfactual donkey sentences as well:

(5) If John owned a donkey, he would beat it.

Representing counterfactuals as $\phi>\psi$ like before, we would like to represent (5) abstractly as $\exists x[P x]>Q x$, while still maintaining the equivalence with $\forall x[P x>Q x]$. The challenge is to account for this equivalence, without giving up our standard dynamic account of indefinites. In van Rooij (2006a) it is shown how this challenge can be met, and I will repeat the argumentation from that paper here. ${ }^{9}$

Suppose that we want to interpret a sentence of the form $\exists x \phi>\psi$ in possibility $\langle w, g\rangle$. According to the standard Lewis/Stalnaker analysis of counterfactuals, we should then select among those possibilities that verify $\exists x \phi$ the ones that are closest to $\langle w, g\rangle$ and check whether they also make $\psi$ true. Because $\phi$ might contain free variables that should be interpreted by means of $g$, the natural context of interpretation of $\exists x \phi$ is the set $W(g)=\{\langle v, h\rangle: v \in W \& h=g\} .{ }^{10}$ After the interpretation of $\exists x P x$, for instance, we end up with a set of world-assignment pairs like

\footnotetext{
8 I assume here that assignments are partial functions.

9 This treatment of counterfactual donkey sentences was crucially inspired by Alonso-Ovalle's (2005) analysis of counterfactuals with disjunctive antecedents.

10 For simplicity we can assume that all worlds have the same domain, but this is quite inessential, because we could also make use of counterpart functions from individuals and worlds to individuals. It is implicitly assumed that $W$ denotes the set of all worlds. This need not always be the case, though.
} 
$\langle v, h\rangle$, where variable $x$ is in the domain of assignment $h$, and $h(x)$ is an element of the set denoted by $P$ in world $v$. Let us denote this set of world-assignment pairs by $/ \exists x P x / g$. To check whether $\exists x[P x]>Q x$ is true in $\langle w, g\rangle$ we have to select among the possibilities in $/ \exists x P x / g$ those that are closest to $\langle w, g\rangle$, and see whether they also verify $Q x$. But this means that we need an ordering relation, $\leq_{\langle w, q\rangle}^{*}$, between worldassignment pairs with respect to possibility $\langle w, g\rangle:\langle u, k\rangle \leq_{\langle w, g\rangle}^{*}\langle v, h\rangle$. Fortunately, there is a natural way to define an ordering ' $\leq^{*}$, between world-assignment pairs in terms of the ordering relation between worlds used by Lewis and Stalnaker: ${ }^{11}$

Definition 1 Given a Lewis/Stalnaker similarity relation $\leq_{w}$ between worlds, we define the similarity relation $\leq_{\langle w, g\rangle}^{*}$ between world-assignment pairs as follows:

$$
\langle v, h\rangle \leq_{\langle w, g\rangle}^{*}\langle u, k\rangle \operatorname{iff}_{\text {def }} h=k \supseteq g \text { and } v \leq_{w} u .
$$

Notice, first, that in case the antecedent $\phi$ of a counterfactual doesn't introduce new variables, or discourse markers, all the elements of $/ \phi / g$ are world-assignment pairs with assignment $g$. Thus, in that case ' $\leq$,' comes down to ' $\leq$ ', because we can now ignore the assignment function. But suppose that $\phi$ is of the form $\exists x P x$. In that case, all the assignments in $/ \exists x P x / g$ differ from $g$ in that they also assign an object to $x$. Let $\langle v, h\rangle$ and $\langle u, k\rangle$ be two possibilities in $/ \exists x P x / g$. According to definition 1 , to check whether one of these is more similar to $\langle w, g\rangle$ than the other only makes sense in case $h$ assigns the same individual to $x$ as $k$ does, $h(x)=k(x) .{ }^{12}$ But this means that we must check for each individual $d$ separately what are the closest possibilities to $\langle w, g\rangle$ that make $P x$ true. We define the selection function as follows:

Definition 2 Given a similarity relation $\leq_{\langle w, g\rangle}^{*}$ between world-assignment pairs as defined in definition 1 and a standard dynamic update function [·], we define the selection function $f^{*}$ from sets of world-assignment pairs to sets of world-assignment pairs as follows:

$$
f_{\langle w, g\rangle}^{*}(/ \phi / g) \stackrel{\operatorname{def}}{=}\left\{\langle v, h\rangle \in / \phi / g \mid \neg \exists\langle u, k\rangle \in / \phi / g,\langle u, k\rangle<_{\langle w, g\rangle}^{*}\langle v, h\rangle\right\},
$$

where $/ \phi / g \stackrel{\text { def }}{=}[\phi](\{\langle v, h\rangle: v \in W \& h=g\})$, and $j<_{i}^{*} k \operatorname{iff}_{\text {def }} j \leq_{i}^{*} k$ but not $k \leq_{i}^{*} j$.

It is easy to see that it now follows that $f_{\langle w, g\rangle}^{*}(/ \exists x P x / g)$ comes out to be equivalent with $\bigcup_{d \in D} f_{\langle w, g\rangle}^{*}\left(\left\{\langle v, g[x / d]\rangle: d \in I_{v}(P)\right\}\right)$. On our assumption that $\phi>\psi$ is true in $\langle w, g\rangle$ iff $\forall\langle v, h\rangle \in f_{\langle w, g\rangle}^{*}(/ \phi / g):\langle v, h\rangle$ verifies $\psi$, we end up with the happy result that $\exists x[P x]>Q x$ is equivalent with $\forall x[P x>Q x] .{ }^{13}$ I conclude that we can account for counterfactual donkey sentences in a natural and compositional way.

\footnotetext{
11 The same ordering has been used in Schulz and van Rooij (2006) to account, for instance, for the intuition that the answer 'John came, Mary came, or both came' to the question 'Who came?' does not give rise to an inconsistency, although it should be interpreted in a global exhaustive way.

12 Though the relation ' $\leq_{w}$ ' is connected, ' $\leq_{\langle w, q\rangle}^{*}$ ' is not.

13 To be sure, this equivalence holds for many-ary donkey sentences as well: if $\vec{x}$ is an $n$-ary tuple of variables and $\phi$ and $\psi$ are $n$-ary predicates, our analysis predicts that $\exists \vec{x}[\phi(\vec{x})]>\psi(\vec{x})$ is equivalent with $\forall \vec{x}[\phi(\vec{x})>\psi(\vec{x})]$.
} 


\section{Universal any as a 'counterfactual' donkey}

Notice that for $\exists x[P x]>Q x$ to be true in world $w$ where $P$ has a non-empty extension it is not enough that all individuals in the extension of $P$ also have property $Q$ : it must also be the case for all non- $P$ individuals that they would have property $Q$ if they had property $P$. Thus, $\exists x[P x]>Q x$ is stronger than $\forall x[P x \rightarrow Q x]$. This last feature suggests that we can account for the extra 'that-can't-be-anaccident'-interpretation of U-any when we think of it as a 'counterfactual' donkey in disguise.

The idea is to translate Dayal's any in a (dynamic) Montague-style framework (with connective ' $>$ ') by ' $\lambda P \lambda Q \exists x[P x]>Q x$ '. ${ }^{14,15}$ Notice, first, that on such an analysis, (1a), Any owl hunts mice, is interpreted as a kind of generic statement, though without exceptions, and that on this analysis (1b), Any farmer who owns a donkey beats it, is treated as a standard (though 'counterfactual') donkey sentence, without any further problems. Second, on this analysis (4a), Any student in Mary's class is working on NPIs, has a stronger meaning than (4b), Every student in Mary's class is working on NPIs, because (4a) has the extra 'that-can't-be-an-accident'-interpretation. ${ }^{16}$ Remember that to account for this effect, we don't have to quantify over possible individuals.

A problem for any analysis of any is that it should be able to account for the fact that whereas (6a) is not appropriate, $(6 b)$ is:
a. *Yesterday, John talked to any woman.
b. Yesterday, John talked to any woman he saw (yesterday).

What has to be explained is how it can be that the extra 'he saw (yesterday)' added to the restrictor of any turns the inappropriate (6a) into the appropriate (6b). According to Dayal (1998), (6a) is inappropriate because it gives rise to too strong truth conditions. Her analysis involves the proposal that $U$-any universally quantifies over possible instead of actual individuals. We don't quantify over possible individuals but can still account for the inappropriateness of (6a) by our 'counterfactual' treatment of any: (6a) would be interpreted as saying that 'for all individuals, if he/she were a woman, John would have talked to him/her yesterday.' According to our analysis, this can only be the case if John talked yesterday not only to all existing women, but also to every non-existing woman, which is an obviously false claim. For this reason, I propose, (6a) is inappropriate. This is all fine, but why,

\footnotetext{
14 Our earlier claim that $\exists x[P x]>Q x$ is equivalent with $\forall x[P x>Q x]$ was based on the implicit assumption that every world has the same domain. In case we don't make that assumption, the equivalence only holds if the universal quantifier quantifies over possible individuals. This shows that our proposal is indeed close to Dayal's analysis.

15 Independently, Kim and Kaufman (2006) proposed to treat some Korean Indeterminate- $\mathrm{Na}$ items in terms of a similarity relation between worlds as well. It would be interesting to see how the proposals relate to each other.

16 The difference between the meaning of any and every disappears, on our analysis, if the restricting noun of the quantifier is 'empty'. Thus, Anybody smokes is predicted to mean the same as Everybody smokes. I take it that the former sentence is less appropriate than the latter, and would like to account for that by noticing that there is a more standard alternative (the sentence with every) with the same meaning.
} 
then, is (6b) suddenly appropriate? Dayal (1998) argues that this is due to the episodic character of the extra 'he saw (yesterday)' added to the restrictor. Again, we will follow Dayal. If we assume that the extra material 'he saw yesterday' should be interpreted in the actual world, ${ }^{17}$ this means that any in (6b) now quantifies only over individuals John actually saw (yesterday). As a result, (6b) will be interpreted as saying that 'for all individuals John actually saw (yesterday), if he/she were a woman, John would have talked to him/her yesterday'. ${ }^{18}$ But then, (6b) becomes a statement with very modest truth conditions, ${ }^{19}$ and there is no pragmatic reason anymore to deem it inappropriate.

In this section we have dismissed K\&L's analysis of U-any as a generic indefinite. This doesn't mean, however, that I would like to give up their constraint on appropriate use of U-any as well. In fact, adopting their constraint gives rise to an interesting consequence. Recall that K\&L propose that any can be used appropriately only if domain widening results in a stronger assertion. Let us say that $\exists x_{D}[P x]$ means that the domain of quantification of ' $\exists$ ' is restricted to individuals in $D$. Observe now that if $D^{\prime} \subset D$, it immediately follows that $\exists x_{D}[P x]>q$ is stronger than $\exists x_{D^{\prime}}[P x]>q$. This is so because $\exists x_{\left\{d_{1}, d_{2}\right\}}[P x]>q$, for example, comes out as being equivalent with $\left(P\left(d_{1}^{*}\right)>q\right) \wedge\left(P\left(d_{2}^{*}\right)>q\right),{ }^{20}$ while $\exists x_{\left\{d_{1}\right\}}[P x]>q$ is equivalent with $P\left(d_{1}^{*}\right)>q$, and is thus weaker. Thus, it is predicted that U-any is appropriate in positive contexts. If we embed U-any under negation, however, domain extension leads to a weaker assertion, ${ }^{21}$ which means that U-any is predicted to be appropriate only in positive contexts. I believe that this is in accordance with the facts. ${ }^{22}$

Let us remind ourselves what we have done in this section: we have accounted for Dayal's observations (or at least the ones mentioned in this paper) concerning U-any without having to give up the popular assumption that all uses of any should be handled by means of existential quantification. Although U-any is represented in terms of existential quantification, we still predict a universal reading and-via K\&L's domain widening and strengthening-that U-any is appropriate only in positive contexts. The picture that emerges, then, is the following: any should always be represented in terms of an existential quantifier, and its use is appropriate just in case domain extension gives rise to a stronger assertion. For NPI-any we follow $\mathrm{K} \& \mathrm{~L}$ and treat it standardly as $\lambda P \lambda Q \exists x[P x \wedge Q x]$, which means that it can be used appropriately (almost) only in DE contexts. For U-any we proposed the represention $\lambda P \lambda Q \exists x[P x]>Q x$, and we have seen that this results in the appropriateness condition that it can be used in positive contexts only.

\footnotetext{
17 Accounted for either by a two-dimensional actuality operator or by a two-sorted logical language that allows for quantification over worlds in the object language. See also Chierchia (2006).

18 Thanks to a reviewer for pointing out a problem with an earlier, slightly different proposal.

19 Though it still has a stronger meaning than the universal 'Yesterday, John talked to every woman he saw.'

${ }^{20}$ Where $d^{*}$ is a constant in the language that uniquely denotes $d$.

${ }^{21}$ This is obvious, because $\neg\left(\exists x_{\left\{d_{1}, d_{2}\right\}}[P x]>q\right)$ is now equivalent with $\neg\left(P\left(d_{1}^{*}\right)>q\right) \vee \neg\left(P\left(d_{2}^{*}\right)>q\right)$, which is weaker than $\neg\left(P\left(d_{1}^{*}\right)>q\right)$, which is equivalent with $\neg\left(\exists x_{\left\{d_{1}\right\}}[P x]>q\right)$.

22 The same prediction is made, of course, by anyone who accounts for U-any in terms of universal quantification like Dayal $(1998,2004)$ and Saeboe (2001).
} 


\section{NPI-any as a weak 'counterfactual' donkey}

Although I find this emerging analysis appealing, it should be clear that we can't be fully satisfied yet. First of all-as will be discussed in the final section-other authors have proposed a conditional analysis of U-any before, and so it is not clear what is won by our particular treatment making use of the counterfactual connective ' $>$ '. Second, the treatment suggested so far of U-any and NPI-any is not yet uniform enough: the connectives involved in the different uses of any are different, and we still have to admit that any is ambiguous. Without claiming that we can get rid of this ambiguity completely, I would like to propose that the different uses of any are almost identical, if we think of NPI-any from a 'counterfactual' donkey perspective as well. I will argue that the U-and NPI-uses of any are as closely related as the strong and weak readings of 'counterfactual' donkey sentences. In this section I will first present the analysis of weak counterfactual donkey sentences given in van Rooij (2006a), and then propose to treat NPI-any in terms of it.

\subsection{Weak counterfactual donkey sentences}

Although I believe that a counterfactual donkey sentence is mostly equivalent to a formula with wide scope universal quantification, there are (at least) two types of examples where this equivalence seems dubious, or even obviously wrong:

(7) a. If Alex were married to a girl from his class, it would be Sue.

b. If I had a dime in my pocket, I would throw it into the meter.

For (7a) to be true, it doesn't seem to be required that for any individual (e.g. Mary), if that individual were from Alex's class and married to him, it would be Sue. For this paper the second type of example, i.e (7b), is more interesting. The indicative version of this example is of course the standard example that shows that not all donkey sentences give rise to universal readings.

The universal reading of donkey sentences in standard DRT, FCS, and dynamic semantics depends on the assumption of unselective binding. I made that assumption in Sects. 3 and 4 as well, by defining the ordering relation between worldassignment pairs as in definition 1. However, this gives rise to the problem of how we can account for weak readings of donkey sentences (the indicative version of (7b)) and for asymmetric readings of adverbs of quantification (the proportion problem). The standard way to solve those problem in dynamic semantics (going back to Root 1986 and also defended in Dekker 1993) is to give up unselective binding for all variables involved. The idea is to quantify not over individual assignments, but rather over equivalence classes of assignment fuctions, and require that for the donkey sentence to be true there should be an element of each equivalence class of assignments verifying the antecedent that makes the consequent true. Two different assignments are in the same equivalence class of assignment functions iff the variables with different values are not being selected over. The nice thing about this solution is that (i) one still treats all indefinites in the 
same way, and (ii) the indefinite whose introduced variable is not unselectively bound can still be picked up anaphorically in the consequent.

So, how does this work for counterfactual donkey sentences? Well, we will represent a counterfactual in general by a formula $\phi>^{X} \psi$, where $\phi$ and $\psi$ are as expected, and $X$ is the set of variables introduced by $\phi$ that is unselectively bound. Notice that even if $\phi$ contains an indefinite, $X$ might still be the empty set. Now let us slightly redefine the ordering relation between possibilities as follows:

Definition 3 Given a Lewis/Stalnaker similarity relation $\leq_{w}$ between worlds, we define the similarity relation $\leq_{\langle w, g\rangle}^{*, X}$ between world-assignment pairs as follows: $\langle v, h\rangle \leq_{\langle w, g\rangle}^{*, X}\langle u, k\rangle \operatorname{iff}_{d e f} h, k \supseteq g, h \uparrow^{X}=k \uparrow^{X}$, and $v \leq_{w} u$.

Here $h \uparrow^{X}$ denotes the restriction of $h$ to $X$, and thus conveys that $h \uparrow^{X}=k \uparrow^{X}$ iff $\forall x \in X: h(x)=k(x)$. What this definition comes down to is a weakening of definition 1 , because it now allows for a comparison between possibilities where the assignments are not the same. In particular, if $X=\emptyset$ it immediately holds that the assignments are irrelevant for the ordering relation: $\langle v, h\rangle \leq_{\langle w, g\rangle}^{*, \emptyset}\langle u, k\rangle$ iff $v \leq_{w} u$.

If one makes the assumption that one can only be married to one girl, this small, but independently motivated, change already accounts for example (7a) above, without making the assumption that indefinites are ambiguous. We redefine the selection function as follows:

Definition $4 f_{\langle w, g\rangle}^{*, X}(/ \phi / g)=\left\{i \in / \phi / g: \neg \exists j \in / \phi / g: j<_{\langle w, g\rangle}^{*, X} i\right\}$.

Now example (7a) is predicted to be true if represented such that $X=\emptyset$ just in case Alex is married to Sue (and only Sue) in the world(s) closest to the actual one where Alex is married to a(ny) girl from his class.

But what should we do to account for counterfactual variants of weak donkey sentences like (7b)? To account for weak readings of counterfactual donkey sentences, we have to assume that there are possibilities closest to the actual world where I have more than one dime in my pocket. What is required to account for such cases is to lump together all of the possibilities where the difference in assignment doesn't matter, and say that only one of those assignments has to be taken into account for the interpretation of the consequent. Let us first say that $\langle v, h\rangle \sim^{X}\langle u, k\rangle$ iff $v=u$ and $h \uparrow^{X}=k \uparrow^{X}$. Then we give the following general truth conditions of conditionals:

Definition $5 \phi\rangle^{X} \psi$ is true in $\langle w, g\rangle$ iff $\forall\langle v, h\rangle \in f_{\langle w, g\rangle}^{*, X}(/ \phi / g): \exists\langle u, k\rangle \in$ $f_{\langle w, g\rangle}^{*, X}(/ \phi / g):\langle u, k\rangle \sim^{X}\langle v, h\rangle$ and $\langle u, k\rangle$ verifies $\psi$.

Notice that with this definition we can account for both strong and weak counterfactual donkey sentences. A strong counterfactual donkey sentence like (5) will be represented by $\exists x[P x]>{ }^{\{x\}} Q x$ and its meaning will be equivalent with that of the universal sentence $\forall x[P x>Q x]$ just as in the original account proposed in Sect. 3.3. A weak counterfactual donkey sentence like (7b), on the other hand, will be represented by a formula of the form $\exists x[P x]>^{\emptyset} Q x$ and is true even if in the closest 
counterfactual world(s) where I have more than one dime in my pocket, I throw only one of them into the meter.

\subsection{NPI-any revisited}

As suggested above, I propose that NPI-any should be treated as a weak 'counterfactual' donkey sentence. More in particular, the proposed (Montague-style) meaning of NPI-any will be $\lambda P \lambda Q \exists x[P x]>^{\emptyset} Q x$. I will show in a moment that given our interpretation of ' $>$ ' , this formula gives rise to the same truth conditions as the more standard $\exists x[P x \wedge Q x]$ if we make one more assumption: that a sentence in which any with restrictor $P$ occurs presupposes that $P$ has a non-empty extension (in the world of interpretation). Notice, first, that this assumption is quite innocent for uses of U-any if these are analyzed as we proposed in Sect.4. In particular, it doesn't exclude at all that we take into account non-actual worlds to verify the sentence in which it occurs. Given our analysis of weak counterfactual donkey sentences and our proposed representation of NPI-any, on the other hand, this innocent looking presupposition has the very welcome effect that $\exists x[P x]>^{\emptyset} Q x$ will have exactly the same truth conditions as the formula $\exists x[P x \wedge Q x]$, which is the standard translation of indefinites. ${ }^{23}$ By K\&L's widening and strengthening analysis, this will thus have the effect that we predict: that NPI-any is, indeed, licensed almost only in DE contexts.

Let me now show why $\exists x[P x]>^{\emptyset} Q x$ will have exactly the same truth conditions as the formula $\exists x[P x \wedge Q x]$, if $P$ has a non-empty extension in the actual world. The first thing to notice is that because the set of variables which NPI-any unselectively quantifies over is empty, i.e. $X=\emptyset$, the ordering relation in terms of which our formula $\exists x[P x]>^{\emptyset} Q x$ is interpreted comes down to the standard Lewis/Stalnaker ordering between possible worlds. With Lewis and Stalnaker we have assumed that this ordering satisfies strong centering, which means that for any two different worlds $v$ and $w, w$ is always strictly more similar to itself than $v$ is to $w: w<_{w} v$. Assuming that $P$ has a non-empty extension in actual world $w$-i.e., assuming that the presupposition is satisfied - it follows by definition 5 that to verify $\exists x[P x]>^{\emptyset} Q x$ we only have to consider the actual world $w$. Take the possibility in which the sentence is interpreted to be $\langle w, g\rangle \cdot{ }^{24}$ By definition 5 again, for $\exists x[P x]>^{\emptyset} Q x$ to be true in $\langle w, g\rangle$, it is enough if there is a $h$ such that $\langle w, h\rangle \in / \exists x P x / g$ and $\langle w, h\rangle$ verifies $Q x$. But this means that there must be a $d \in D$ such that $h=g\left[{ }^{x} / d\right]$ (meaning as usual that $h$ is just like $g$ except that it assigns variable $x$ to individual $d$ ) and $h(x) \in I_{w}(P)$ and $h(x) \in I_{w}(Q)$ (where $I_{w}(P)$ denotes the extension of $P$ in $w$ according to the model's interpretation function $I)$. But that obviously means that $\exists x[P x]>^{\emptyset} Q x$ has exactly the same truth conditions as the formula $\exists x[P x \wedge Q x]$, if $P$ has a non-empty extension, which is precisely what we wanted to show.

Given this proof, we have shown that we can treat not only U-any as a 'counterfactual' donkey sentence (in disguise), but NPI-any as well. The only difference

\footnotetext{
23 One might complain that in this way we can't account for sentences like There isn't any unicorn in the garden. I don't think this is a serious problem, because I find this sentence appropriate only in case it is assumed (for the sake of argument) that there are unicorns.

24 But notice that the identity of the assignment is quite irrelevant.
} 
is that whereas U-any is interpreted as a strong donkey sentence, NPI-any is interpreted as a weak donkey sentence. Exactly because of this, (i) U-any gets a universal interpretation and NPI-any an existential one, and thus (ii) U-any is via K\&L's analysis licensed only in positive contexts, while NPI-any is licensed (almost) only in negative contexts. ${ }^{25}$

I conclude that an analysis of the different uses of any as 'counterfactual' donkey sentences (in disguise) is quite successful because (i) it gives rise to (approximately) the same meanings as the successful analyses of U-any (Dayal) and NPI-any $(\mathrm{K} \& \mathrm{~L})$, but it allows us to treat them all in an (almost) uniform way, and reduce their differences to an independently required distinction between different readings of donkey sentences (strong versus weak). ${ }^{26}$

\section{Free choice any}

In the previous section we have accounted for 'Universal' and 'NPI'-any in a uniform way. We have not yet explained how the following free choice reading of any should be accounted for:

You may take any card.

The main challenge is, of course, to account for the universal reading of (8). Given that U-any gives rise to a universal reading, but NPI-any does not, it seems reasonable to think of (8) in terms of U-any. The problem is that in order to get the universal reading, we have to scope the existential quantifier over the modality and represent (8) as $\exists x C x>^{\{x\}}$ May $(j, T x)$. Although this representation gives us the intuitively correct reading, ${ }^{27} \mathrm{I}$ believe the price of this unusual scoping is too high. If we want to give 'may' scope over the existential quantifier, however, and represent

${ }^{25}$ Rooth (1987) observed that some quantified donkey sentences (e.g. ones with 'every') typically give rise to strong readings, whereas others (e.g. ones with 'no' or 'few') typically give rise (only) to weak readings. Is this a problem for the proposed analysis (as one reviewer wondered)? Well, we predict that any in examples like 'No/few officer(s) gave any member of a squad its orders' should be analyzed as a weak counterfactual donkey, because the item occurs in a DE context. I think this is in accordance with intuition.

${ }^{26}$ Our analysis predicts that the following comparatives with any are appropriate:

(i) Mary is taller than any man.

(ii) Any man is taller than Mary.

On Seuren's (1973) analysis of comparatives, John is taller than Mary iff $\exists d[\operatorname{Tall}(j, d) \wedge \neg \operatorname{Tall}(m, d)]$, where $d$ is a degree and 'Tall' a relation between individuals and degrees. On this analysis, any occurs in a DE context in (i) and in a positive context in (ii). Applying K\&L's appropriateness condition, this means that we have to represent (i) by a formula like $\exists d\left[\operatorname{Tall}(m, d) \wedge \neg\left(\exists x M x>^{\emptyset} \operatorname{Tall}(x, d)\right)\right]$, while (ii) should be represented either by $\exists d[(\exists x M x>\{x\} \operatorname{Tall}(x, d)) \wedge \neg \operatorname{Tall}(m, d)]$ or by $\exists x M x>\{x\}(\exists d$ $[\operatorname{Tall}(x, d) \wedge \neg \operatorname{Tall}(m, d)])$. If we make the natural assumption that Tallness is monotone decreasing in degrees $\left(\forall x, d, d^{\prime}\left[\left(\operatorname{Tall}(x, d) \wedge d^{\prime}<d\right) \rightarrow \operatorname{Tall}\left(x, d^{\prime}\right)\right]\right)$ the latter two analyses are identical. Something similar happens with von Stechow's (1984) analysis of comparatives.

${ }^{27}$ If we assume that the ordering between worlds required to analyze the conditional connective ' $>$ ' is such that $v<_{w} u$ iff $I(C)(w) \subseteq I(C)(v) \subset I(C)(u)$, we make the same predictions as the system proposed by Menendez-Benito (2005), who makes use of universal quantification and an exclusiveness operator. See Sect. 7 for more discussion. 
(8) by May $\left(j, \exists x C x>>^{\{x\}} T x\right)$, we don't receive the correct interpretation. This suggests that we should represent the any in (8) in terms of ' $>$ ' instead of ' $>\{x\}$ '. Now the problem is that on a standard analysis of the modal, we can't derive the universal reading. ${ }^{28}$ I will suggest that this is due to a wrong choice of interpretation of 'may' in (8). I will show that if we analyze the permission performatively, as originally proposed by Lewis (1970/1919) and Kamp (1979), but account for this performative analysis in a new distributive way, we can derive the universal reading of (8) and can explain why domain widening gives rise to a stronger reading.

According to the performative account, if the master commands John to do $\phi$ by saying 'You must do $\phi$ ', or allows John to do $\phi$ by saying 'You may do $\phi$ ', it is typically not yet the case that the proposition expressed by $\phi$ is respectively a superset of, or consistent with, John's permissibility set, $\Pi$, represented by a set of possible worlds. However, the performative effect of the command/permission will be such that in the new context what is commanded is a superset of, and what is permitted is consistent with, the new permissibility set. Thus, in case the command or permission is not used vacuously, the permissibility set, $\Pi^{\prime}$, of the new context will be different from $\Pi$, so that the obligation/permission sentence will be satisfied. Our problem is to say how command and permission sentences govern the change from the prior permissibility set, $\Pi$, to the posterior one, $\Pi^{\prime}$.

Kamp (1979) proposed to solve this problem by assuming that the change from prior to posterior permission set is governed by (what is now called) revision and contraction. The idea is that we not only have a set of best, or ideal, worlds, but also a global reprehensibility ordering that says which non-ideal worlds are better than others. It is well known that such an analysis can account for the free choice effect of permission sentences like 'You may take an apple or a pear', but only in case taking apples and taking pears is equally reprehensible. Although this proposal is very natural, it is not quite good enough to account for the free choice effect of 'You may take any apple' (if 'any' is represented by an existential quantifier). On Kamp's performative analysis one can only conclude from this sentence that one is allowed to take apple $a$ iff apple $a$ is among the least reprehensible ones to take. The free choice effect seems to be stronger, though, and independent of a reprehensibility ordering. Van Rooij (2006a) proposed to tackle this problem by assuming that the change from prior to posterior permission state is sensitive to the assignments as well. Here we will follow a recent suggestion by Schulz (2008) to account for the update effect of permissions in terms of a local instead of a global ordering relation between worlds. The local ordering relation is the standard one used in premise semantics: given a set of formulas $\Psi$, we define $v$ to be at least as 'good' as $w$, $w \leq_{\Psi} v$, iff all formulas in $\Psi$ true in $w$ are also true in $v$.

$$
w \leq_{\Psi} v \quad \text { iff }_{\text {def }} \quad \forall \psi \in \Psi: w \models \psi \Rightarrow v \models \psi
$$

\footnotetext{
28 Quite a number of authors (e.g. Kratzer and Shimoyama 2002; Schulz 2005, Fox 2006) have proposed to account for the 'conjunctive' implication of a sentence like 'You may take an apple or a pear' in terms of conversational implicatures. Thus one might propose (e.g. Chierchia 2006; Aloni and van Rooij 2007) to account for the universal reading of (8) by means of a conversational implicature as well. However, conversational implicatures are cancelable, and the universal reading of (8) is not, which suggests (to me) that the universal reading of this sentence should not be accounted for by a Gricean implicature after all.
} 
In terms of this ordering relation, we now define a function that assigns to a world $w$ and a formula $\phi$ the $\phi$-worlds which are minimal extensions of $w$ :

$$
f_{\leq}(w, \phi) \stackrel{\text { def }}{=}\{v \in \llbracket \phi \rrbracket: w \leq v \& \neg \exists u \in \llbracket \phi \rrbracket: w \leq u \& u<v\}
$$

The idea is that if we previously were in allowed world $w$ and are now allowed to make $\phi$ true, we are allowed to go to any $\phi$-worlds that are minimal extensions of $w$. As in standard dynamic semantics, we assume that the (performative) effect of a permission sentence is modeled as a function from prior to posterior permission set. In fact, following Schulz (2008) we will make use of a partial semantics here. The positive update function of a permission with respect to permission set $\Pi$ is defined by means of this choice function given above:

$$
\Pi[\operatorname{May} \phi]^{+} \stackrel{\operatorname{def}}{=} \Pi \cup \bigcup_{w \in \Pi} f(w, \phi)
$$

Thus, the result of the permission is that new worlds are added to the prior permission set. The negative update of the same sentence does the reverse: it eliminates $\phi$-worlds from the permission set.

$$
\Pi[\operatorname{May} \phi]^{-} \stackrel{\operatorname{def}}{=} \Pi-\bigcup_{w \in \Pi} f(w, \phi)
$$

This definition of the positive and negative update functions wouldn't be complete without a recursive definition of the meaning of the sentential connectives. This recursive definition is given below:

$$
\begin{aligned}
& \Pi[\neg \phi]^{+} \stackrel{\operatorname{def}}{=} \Pi[\phi]^{-} \quad \Pi[\neg \phi]^{-} \stackrel{\operatorname{def}}{=} \Pi[\phi]^{+} \\
& \Pi[\phi \wedge \psi]^{+} \stackrel{\operatorname{def}}{=} \Pi[\phi]^{+}[\psi]^{+} \quad \Pi[\phi \wedge \psi]^{-} \stackrel{\operatorname{def}}{=} \Pi[\phi]^{-} \cup \Pi[\psi]^{-}
\end{aligned}
$$

If we now define ' $\vee$ ' in terms of ' $\neg$ ' and ' $\wedge$ ' as usual, and also define the update with 'Must' in terms of the update with 'May' in the standard way:

$$
\Pi[\text { Must } \phi]^{+} \stackrel{\text { def }}{=} \Pi[\neg \text { May } \neg \phi]^{+} \quad \Pi[\text { Must } \phi]^{-} \stackrel{\text { def }}{=} \Pi[\neg \text { May } \neg \phi]^{-},
$$

we receive the appealing result that $\Pi[\text { Must } \phi]^{+}=\Pi \cap \llbracket \phi \rrbracket$.

To account for entailments between permission sentences, Kamp (1979) proposed the notion of $p$-entailment. It says that 'May $\psi$ ' follows from 'May $\phi$ ' $\left.\operatorname{May}(j, \phi) \models^{p} \operatorname{May}(j, \psi)\right)$ iff adding the permission that $\psi$ doesn't change the permission set anymore after you were permitted to do $\phi$. On our new dynamic analysis, this means that the permission of $\psi$ follows from the permission of $\phi$ iff $_{\text {def }}$ for all permission states, $\Pi: \Pi[\phi]^{+}=\Pi[\phi]^{+}[\psi]^{+}$. Notice that in case $Q(a)$ and $Q(b)$ are elements of the set $\Psi$ in terms of which the ordering relation is defined, it follows that $\operatorname{May}(j, Q(a) \vee Q(b)) \models_{d} \operatorname{May}(j, Q(a)) \wedge \operatorname{May}(j, Q(b))$. If it is also the case that $Q(c)$ is an element of $\Psi, \operatorname{May}(j, Q(c))$ doesn't follow from May $(j, Q(a) \vee Q(b))$. 
However, May $(j, Q(c))$ does follow from May $(j, Q(a) \vee Q(b) \vee Q(c))$. This is crucial for us to account for the universal 'reading' of (8).

We have seen above that on our suggested representation of (8) by $\operatorname{May}\left(j, \exists x P x>^{\emptyset} Q x\right)$, the embedded clause of (8) is truth-conditionally equivalent with $\exists x P x \wedge Q x$ for worlds in $\Pi$ that satisfy the presupposition that predicate $P$ has a non-empty extension. Thinking of this existential sentence as a (large) disjunction, and making use of the above reasoning, we have accounted for the free choice effect of (8). Moreover, on our analysis it follows that the permission to take a(ny) card is strengthened if one extends the domain: $\operatorname{May}\left(j, \exists x_{D} P x\right) \models^{p} \operatorname{May}\left(j, \exists x_{D^{\prime}} P x\right)$ if $D \supset D^{\prime}$. This means that on Kadmon and Landman's analysis of any, we have explained why this item is licensed in permission sentences.

Dayal forcefully argued that any analysis of any has to account for the contrast between the acceptability of (8) versus the unacceptability of (9):

*You must take any card.

On our proposed analysis this follows immediately on the obvious extension of Kamp's notion of $p$-entailment to commands: $\operatorname{Must}(j, \phi) \models^{p} \operatorname{Must}(j, \psi)$ iff adding the obligation that $\psi$ doesn't change the permission set anymore after you were obliged to do $\phi .^{29}$ To see that this follows, notice that the update of $\Pi$ with $\operatorname{Must}\left(j, \exists_{D} Q x\right)$, i.e. $\Pi \cap \llbracket \exists_{D} Q x \rrbracket$, doesn't have to entail the following formula, $\exists_{D^{\prime}} Q x$, where $D^{\prime} \subset D$. For if $D=\{a, b, c\}$, and the worlds in $\Pi$ where John takes cards $a, b$, and $c$ are $w_{1}, w_{2}$, and $w_{3}$, respectively, $\Pi \cap \llbracket \exists_{D} Q x \rrbracket$ will be $\left\{w_{1}, w_{2}, w_{3}\right\}$, while $\Pi \cap \llbracket \exists_{D^{\prime}} Q x \rrbracket$, with $D^{\prime}=\{a, b\} \subset\{a, b, c\}=D$, will be $\left\{w_{1}, w_{2}\right\}$. Thus, after the obligation of $\exists x_{D} Q x$ the permission set will still change when you get the obligation that $\exists x_{D^{\prime}} Q x$. But this means that domain extension does not result in a stronger obligation, which means that K\&L's constraint for the appropriate use of any is not met. ${ }^{30}$

\section{Comparison and conclusion}

Negative polarity and free choice items are hotly debated in the literature. But apart from the papers of Dayal and K\&L (and Krifka), I haven't made much reference to this discussion in this paper. Although it is impossible to do justice to all proposed analyses, in this final section I will briefly relate my analysis to at least some other analyses not discussed so far, and lay out some possible modifications and extensions.

\footnotetext{
29 Kamp's notion of $p$-entailment and this extension are both special cases of Veltman's (1996) more general entailment relation between speech acts.

30 As noted by Giannikidou (2001), among others, in direct commands, any can be used appropriately:

(i) Take any card.

Intuitively, (i) means 'You must take a card, it doesn't matter which.' If we would analyze (9) and (i) in the same way, we wouldn't be able to account for the contrast, obviously. I would suggest that (9) is inappropriate but (i) is not because only the latter expresses a command and permission at the same time; the former expresses (or wants to express) just a command.
} 
A prominent analysis of crosslinguistic (variants of) any is proposed in various papers of Anastasia Giannakidou. Like us, she argues for an existential analysis of any, but seeks to account for its appropriateness in terms of licensing conditions in the style of Ladusaw (1979). In Giannakidou (2001), for instance, it is claimed that NPI-any is licensed in non-veridical contexts, while FC-any (including our U-any) also demands that the context be episodic. Giannakidou argues against Ladusaw's analysis with an impressive battery of counterexamples, but at least some of these counterexamples can be accounted for by other approaches that distinguish between the several potential meanings of any (like K\&L and Dayal). I won't discuss these examples here, but just want to note that Giannnakidou as well makes use of a (strict) conditional to account for U-any, following Legrand (1975). Indeed, one can account for the intuition that U-any is stronger than every by treating it in terms of a strict conditional that quantifies over worlds. However, it doesn't seem to be possible - in contrast to our analysis in terms of counterfactual connective ' $>$ ' - to give a similar treatment of NPI-any in terms of strict conditionals such that this item gives rise to its standard existential meaning. ${ }^{31}$ In this sense, Legrand's and Giannakidou's analyses of any will end up being less uniform than ours.

A semantically uniform analysis of both uses of any has been proposed by Chierchia (2006), modified by Aloni and van Rooij (2007). ${ }^{32}$ Chierchia follows $\mathrm{K} \& \mathrm{~L}$ in their analysis of any as an ordinary indefinite, with reliance on the 'widening-leads-to-strengthening' condition, but seeks to account for U-any by conversational implicature rather than by appealing to genericity. Thinking of an existential sentence as a large disjunction, Chierchia (2006) proposes that ' $A \vee B$ ' gives rise to the conversational implicature that the speaker doesn't know that ' $A \wedge \neg B$ ' is true and doesn't know that ' $\neg A \wedge B$ ' is true. By assuming that the speaker knows what he is talking about, we can conversationally implicate that the speaker knows that both conjunctions are false, and thus that ' $A \wedge \neg B$ ' and ' $\neg A \wedge B$ ' are both false. But in conjunction with the assertion this means that it has to be the case that ' $A \wedge B$ ' is true. Although appealing, I believe (on second thought) that such an approach is problematic, for two reasons. First of all, it does not really account for the 'that-can't-be-an-accident' force of the universal reading. Second, it assumes that the relevant conversational implicatures are uncancelable, which goes against the very idea of something being an implicature in the first place. Most naturally, what can be cancelled is the assumption that the speaker is knowledgeable: the step from 'not know $A$ ' to 'know not $A$ '. Applying cancelability to Chierchia's reasoning, however, would make the wrong prediction: it is predicted that the U-any sentence can be true, although at the same time the speaker doesn't know that the universal statement is true. So, with Jayez and Tovena (2005), I would argue against an analysis that accounts for the universal force of U-any in terms of a conversational implicature.

Where Chierchia wants to derive the strong reading of U-any by making strong knowledge assumptions, Javez and Tovena (2005) and others would rather like to infer universality from one's lack of knowledge, or from one's indifference. In

\footnotetext{
31 That is, without making strong ad hoc assumptions.

32 For a related approach, see Abrusan (2006).
} 
fact, this assumption is not very different from Dayal's (1998) vagueness requirement. Dayal argues that 'John talked to any of these women' is bad because in order for the speaker to know that the sentence is true, the speaker has to know of each of the individual women he is looking at that John talked to her. But this requirement violates Dayal's vagueness constraint on the quantificational domain of U-any that the speaker should not know (or care about, we might add) exactly (roughly speaking) who he was talking about. Part of this irrelevance or lack of knowledge requirement we have already accounted for by our 'counterfactual' analysis: to see whether a sentence of the form ' $\exists x[P x]>^{\{x\}} Q x$ ' is true we can't limit ourselves to the individuals who actually have property $P$. In fact, given that Dayal's treatment of whatever (Dayal 1997) is so close to her analysis of FC-any - and because of Legrand's (1975) claim that free relatives combined with wh-ever are semantically equivalent to any combined with a relative clause-it looks attractive to account for the universal reading of sentences like 'John voted for whoever was on top of the ballot' in terms of a 'counterfactual' donkey analysis as well. ${ }^{33}$ Another part we have not accounted for yet: that the speaker doesn't know, or care about, which individuals actually have property $P$. I don't see any harm being done if we add this constraint as a presupposition for the use of universal (or perhaps of any?) uses of any.

Two final papers I would like to mention are Menenez-Benito (2005) and Aloni (2006). Both assume that occurrences of free choice any (or their Spanish and Italian counterparts) should be interpreted as indeterminate pronouns along the lines of Kratzer and Shimoyama's (2002) analysis of German existential free choice items. According to their proposals, the free choice item introduces a set of (Hamblian) alternative propositions, and the universal interpretation is guaranteed by assuming that at top level we universally quantify over the alternative propositions. Interestingly, they explain why any is appropriate under may but not under must by assuming that the propositional alternatives are mapped into a set of mutually exclusive propositions because of an exclusiveness (Menendez-Benito 2005) or an exhaustivity operator (Aloni 2006). This exclusiveness is consistent with the existential meaning of may, but not with the universal meaning of must. Although neither of these approaches can really account for the 'that-can't-be-anaccident' force of U-any, I think they give rise to empirical predictions very similar to my approach. However, I would like to claim that my proposal depends on (arguably) less controversial assumptions: in contrast to them, I don't need a Hamblin-style semantics for indefinites, propositional alternatives, or a covert universal quantification over propositional alternatives. Most of all, however, their assumption that we need a covert exclusiveness or exhaustivity operator to account for free choice permissions like (8) is not needed, because exclusiveness follows from my treatment: we are looking for minimally different worlds (from a world, or set of worlds, where no card is taken). If (8) is analyzed in terms of U-any $-\exists x C x>{ }^{\{x\}}$ May $T x-$ exclusiveness is due to the meaning of the

\footnotetext{
33 Though following observations of Jacobson (1995) and others, we should perhaps allow the variable to range over pluralities now. This would also account for Choi's observation (p.c.) that you may take more than one thing after the permission 'You may take whatever is given to you.'
} 
counterfactual connective; if (8) is analyzed in terms of NPI-any and a performative analysis, exclusiveness follows from a minimal change of the permission set.

In this paper I have limited myself to the use of the English item any. This is probably still the most discussed word in any discussion of negative polarity and/or free choice items. However, more recently people have become very interested in the issue how negative polarity and free choice items are expressed in languages other than English, and discovered that there exists large variability (see Choi 2007 and Vlachou 2007 for two extensive recent studies). I would have liked to explore what my analysis of any might have to offer on the issue of language variability, but I'm afraid I am not competent enough on these matters to enter into such discussions and leave that to others. I will also leave to others the proposal to take the two any's to be even more look-alike than what is proposed in this paper, by assuming that semantically speaking all donkey sentences have only weak truth conditions, and accounting for the strong readings in terms of pragmatics.

Acknowledgments This paper was presented in Amsterdam, Frankfurt, and Hamburg, and I would like to thank the audiences of these talks and the reviewers for their comments and suggestions. Van Rooij (2006b) is an early version of this paper.

Open Access This article is distributed under the terms of the Creative Commons Attribution Noncommercial License which permits any noncommercial use, distribution, and reproduction in any medium, provided the original author(s) and source are credited.

\section{References}

Abrusan, M. 2006. Even and free choice any in Hungarian. In Proceedings of Sinn und Bedeutung, vol. 11, ed. E. Waldmüller, 1-15. Barcelona: Universitat Pompev Fabra.

Aloni, M. 2006. Free choice and exhaustification: An account of subtrigging effects. In Proceedings of Sinn und Bedeutung, vol. 11, ed. E. Waldmüller, 16-30. Barcelona: Universitat Pompev Fabra.

Aloni, M., and R. van Rooij 2007. Free-choice items and alternatives. In Proceedings of KNAW Colloquium: Cognitive Foundations of Interpretation in 2004, VLN 190, 5-26.

Alonso-Ovalle, L. 2005. Distributing the disjuncts over the modal space. In Proceedings of the North East Linguistics Society, vol. 35, ed. L. Bateman and C. Ussery. Amherst: GLSA.

Chierchia, G. 2006. Broadening your views: Implicatures of domain widening and the "logicality" of language. Linguistic Inquiry 37: 535-590.

Choi, J. 2007. Free choice and negative polarity: A compositional analysis of Korean polarity sensitivity. $\mathrm{PhD}$ Thesis, University of Pennsylvania.

Dayal, V. 1997. Free relatives and ever: Identity and free choice readings. In Proceedings of SALT 7, 72-93.

Dayal, V. 1998. ANY as inherently modal. Linguistics and Philosophy 21: 433-476.

Dayal, V. 2004. The universal force of free choice any. In Linguistic variation yearbook, vol. 4, ed. J. Rooryck, 5-40. Amsterdam: John Benjamin.

Dekker, P. 1993. Transsentential meditations. PhD Thesis, University of Amsterdam.

Fox, D. 2006. Free choice and the theory of scalar implicatures. Ms., MIT.

Giannakidou, A. 2001. The meaning of free choice. Linguistics and Philosophhy 24: 659-735.

Groenendijk, J., and M. Stokhof 1991. Dynamic predicate logic. Linguistics and Philosophy 14: 39-100.

Heim, I. 1982. The semantics of definite and indefinite noun phrases. PhD Diss, University of Massachusetts, Amherst.

Heim, I. 1984. A note on polarity sensitivity and downward entailingness. In Proceedings of the North East Linguistics Society, vol. 14, ed. C. Jones and P. Sells, 98-107. Amherst: GLSA.

Jacobson, P. 1995. On the quantificational force of English free relatives. In Quantification in natural language, ed. E. Bach, et al., 451-486. Dordrecht: Kluwer. 
Jayez, J. and Tovena, L. 2005. When 'widening' is too narrow. In Proceedings of the 15th A'dam Colloquium, ed. P. Dekker and M. Franke, 131-136. Amsterdam: ILLC.

Kadmon, N., and Landman. F. 1993. Any. Linguistics and Philosophy 16: 353-422.

Kamp, H. 1979. Semantics versus pragmatics. In Formal semantics and pragmatics for natural language, ed. F. Guenthner and J. Schmidt, 225-278. Dordrecht: Reidel.

Kamp, H. 1981. A theory of truth and semantic representation. In Formal methods in the study of language, ed. Groenendijk, et al., 277-322. Amsterdam: Mathematise Centrum.

Kim, M., and S. Kaufman. 2006. Domain restriction in freedom of choice. A view from Korean Indet-Na items. In Proceedings of Sinn und Bedeutung, vol. 11, ed. E. Waldmüller, 375-389. Barcelona.

Kratzer, A., and J. Shimoyama 2002. Indeterminete pronouns: The view from Japanese. In Proceedings of 4th Tokyo Conference on Psycholinguistics, 1-15. Tokyo: Hituzisyobo.

Krifka, M. 1995. The semantics and pragmatics of polarity items. Linguistic Analysis 25: 209-258.

Ladusaw, W. A. 1979. Negative polarity as inherent scope. PhD Thesis, University of Texas, Austin.

Legrand, J. 1975. Or and any: The syntax and semantics of two logical operators. PhD diss, University of Chicago.

Lewis, D.K. 1973. Counterfactuals. Oxford: Blackwell.

Lewis, D.K. 1970/1979. A problem about permission. In Essays in honour of Jaakko hintikka. ed. E. Saarinen et al., Dordrecht: Reidel.

Menendez-Benito, P. 2005. The grammar of choice, PhD Thesis, University of Massachusetts, Amherst.

Root, R. 1986. The semantics of anaphora in discourse. PhD Thesis, University of Texas, Austin.

Rooth, M. 1987. Noun phrase interpretation in Montague grammar, file change semantics, and situation semantics. In Generalized quantifiers, ed. P. Gärdenfors, 237-269. Dordrecht: Reidel.

Saeboe, K. J. 2001. The semantics of Scandinavian Free Choice Items. Linguistics and Philosophy 24: 737-787.

Schulz, K. 2005. A pragmatic solution to the paradox of free choice permission. Synthese 147: 343-377.

Schulz, K. 2008. Performative 'may'. Ms., Universiteit van Amsterdam.

Schulz, K., and R. van Rooij. 2006. Pragmatic meaning and non-monotonic reasoning: The case of exhaustive interpretation. Linguistics and Philosophy 29: 205-250.

Seuren, P. 1973. The comparative. In Generative grammar in Europe, ed. F. Kiefer and N. Ruwet, 528-564. Dordrecht: Reidel.

Stalnaker, R.C. 1968. A theory of conditionals. In Studies in logical theory, American philosophical quarterly monograph series, no. 2, ed. N. Rescher, 98-112. Oxford: Blackwell.

van Rooij, R. 2003. Negative polarity items in questions. Journal of Semantics 20: 239-273.

van Rooij, R. 2006a. Free choice counterfactual donkeys. Journal of Semantics 23: 383-402.

van Rooij, R. 2006b. How to donkey FC- and NPI-any. In Proceedings of Sinn und Bedeutung, vol. 11, ed. E. Waldmüller, 479-493. Barcelona.

Veltman, F. 1996. Defaults in update semantics. Journal of Philosophical Logic 25: 221-261.

Vlachou, E. 2007. Free choice in and out of context: Semantics and distribution of French, Greek and English free choice items. PhD Thesis, Universiteit Utrecht, Utrecht.

von Stechow, A. 1984. Comparing semantic theories of comparison. Journal of Semantics 3: 1-77. 